\title{
Simplified Model of a Layer of Interconnects under a Spiral Inductor
}

\author{
Sonia M. Holik, Timothy D. Drysdale \\ Electronics Design Centre, Division of Electronics and Nanoscale Engineering, School of Engineering, University of Glasgow, \\ Glasgow, United Kingdom. \\ Email:sholik@elec.gla.ac.uk, tim.drysdale@glasgow.ac.uk
}

Received February $21^{\text {st }}, 2011$; revised April $2^{\text {nd }}, 2011$; accepted May $10^{\text {th }}, 2011$.

\begin{abstract}
An empirical effective medium approximation that provides a homogeneous equivalent for a layer of interconnects underneath a spiral inductor is presented. When used as part of a numerical $3 D$ model of the inductor, this approach yields a faster simulation that uses less memory, yet still predicts the quality factor and inductance to within $1 \%$. We expect this technique to find use in the electromagnetic modeling of System-on-Chip.
\end{abstract}

Keywords: Effective Medium Theory, Interconnects, Spiral Inductor

\section{Introduction}

On-chip spiral inductors are usually fabricated over areas that are either unused or contain dummy fill and/or patterned ground shields. While fabrication process geometries continue to shrink, inductor sizes are fixed by the usual physical laws. Hence, the use of passive components in System-on-Chip is increasingly inefficient. The under-utilised area underneath inductors could alternatively be used for routing interconnects associated with other circuits (e.g. buses) resulting in significant downscaling of the size of the entire chip. Unfortunately, this presents a challenging electromagnetic modelling problem that is difficult to solve with existing numerical techniques. In particular, the number of features necessary to adequately represent interconnects below an inductor is large due to the small geometrical size of the wires compared to the area occupied by the inductor. Such simulations can be done on isolated structures [1] but they tend to be slow and computationally demanding. The maximum problem size is limited by the available computer memory capacity and computational speed, requiring practical circuit designs to be analysed by splitting into smaller segments first [2]. An improved approach would do away with the need to subdivide the problem. A recently proposed approach [3,4] achieves this by exploiting the large difference in scale between interconnects (microns) and the wavelength (centimetres). Using effective medium theory (EMT), structures that are much smaller than a wavelength (here the intercon- nect) can be replaced in numerical and analytical models by its homogenous equivalent. A key measure of the validity of the EMT approach is that the model continues to accurately predict the behaviour of the whole system considered. The presence of conducting fill underneath an inductor naturally changes the electromagnetic field distribution, and hence the characteristic inductor's parameters. The effect of the dummy metal pattern on the same metal layer as the on-chip inductor [5] and under the spiral [6] with different pattern and configuration of the fill has already been reported. The most significant influence of the metal fill on the inductor performance is the change in the self resonance frequency which has the highest value for an inductor with no fill. Variation in its value is mainly associated with the increase of parasitic capacitance introduced by the metal fill. The concept of applying the EMT to the analysis of stand alone interconnect structures has been numerically and experimentally validated $[3,4]$. In this Paper it is numerically demonstrated that such an approach can be applied to the modelling of passive components, with the eventual aim of assisting designers to reduce chip sizes via improved modelling tools.

\section{Methodology and Homogenisation Procedure}

A numerical model of an example inductor was built and solved using HFSS software [7]. The model was based on standard conventional CMOS technology [8]. The 
geometry of the model is shown in Figure 1, with the top layout view of 7.5 turn rectangular spiral inductor of inner diameter $\alpha=100 \mu \mathrm{m}$, width $10 \mu \mathrm{m}$ and spacing $2 \mu \mathrm{m}$ in Figure 1(a), a perspective view of the spiral inductor with metal rods aligned in parallel underneath the inductor in Figure 1(b), and a cross section of the structure in Figure 1(c). The inductor is defined in the topmost metal, Metal 3, a $1.1 \mu \mathrm{m}$ thick layer of low conductivity aluminium $\sigma=30 \times 10^{6} \mathrm{~S} / \mathrm{m}$. The underpass at Metal 2 has thickness $0.74 \mu \mathrm{m}$. The isolating silicon-oxide layer between Metal 2 and Metal 3 is $0.8 \mu \mathrm{m}$ thick, while between Metal 2 and Metal 1, and Metal 1 and the substrate, it is $1 \mu \mathrm{m}$ thick. The interconnects are placed on Metal 1 , and are defined as floated metal (copper) rods, i.e. they are not connected to ground. The rods are arranged in parallel, and have length $300 \mu \mathrm{m}$ in order to cover the entire span of the inductor. The rods have width $0.45 \mu \mathrm{m}$ and height $0.9 \mu \mathrm{m}$ giving an aspect ratio of 2 . The space between the rods is $0.45 \mu \mathrm{m}$, giving a metal fill factor of 0.5 . The rods and inductor were modelled using layered impedance boundary conditions. The substrate is defined as $15 \Omega-\mathrm{cm}$ resistivity silicon of thickness $625 \mu \mathrm{m}$ and is grounded by the perfect electric boundary conditions defined at the bottom plane, while other walls were defined as radiation boundaries. The spiral was covered by a $60 \mathrm{~nm}$ thick silicon-nitrate passivation layer.

A modified Maxwell-Garnett mixing rule $[3,4]$ is used to calculate the effective permittivity of the homogeneous equivalent layer

$$
\varepsilon_{e f f}=\varepsilon_{e}+\Psi f \varepsilon_{e} \frac{\varepsilon_{i}-\varepsilon_{e}}{\varepsilon_{i}+2 \varepsilon_{e}-f\left(\varepsilon_{i}-\varepsilon_{e}\right)},
$$

where $\varepsilon_{i}$ and $\varepsilon_{e}$ are the dielectric functions of the inclusion and host material respectively (here, a metal and a dielectric), $\Psi$ is a constant relating the fields inside and outside the inclusions (typically $\Psi=3$ for spherical inclusions), $f$ is the filling factor or ratio of the volume of the inclusion to the total size of the unit cell. The Maxwell-Garnett rule is known to give a qualitatively correct prediction of the effective properties of a composite with conducting inclusions [9]. The frequency dependant dielectric function of a metal inclusion $\varepsilon_{i}$ is expressed by a Drude model.

\section{Results and Discussion}

First, it was verified that placing metal rods underneath the spiral inductor influenced its electrical characteristics. Figure 2 illustrates the change in the value of quality factor when there are 20 and 50 metal rods below the spiral with respect to the reference structure without metal rods in the vicinity. The reference structure has inductance of $16 \mathrm{nH}$, a maximum Q-factor of 3.04 at $1.1 \mathrm{GHz}$, and a self-resonant frequency (SRF) of $5.1 \mathrm{GHz}$. When 20 (50) metal rods were added underneath the inductor,
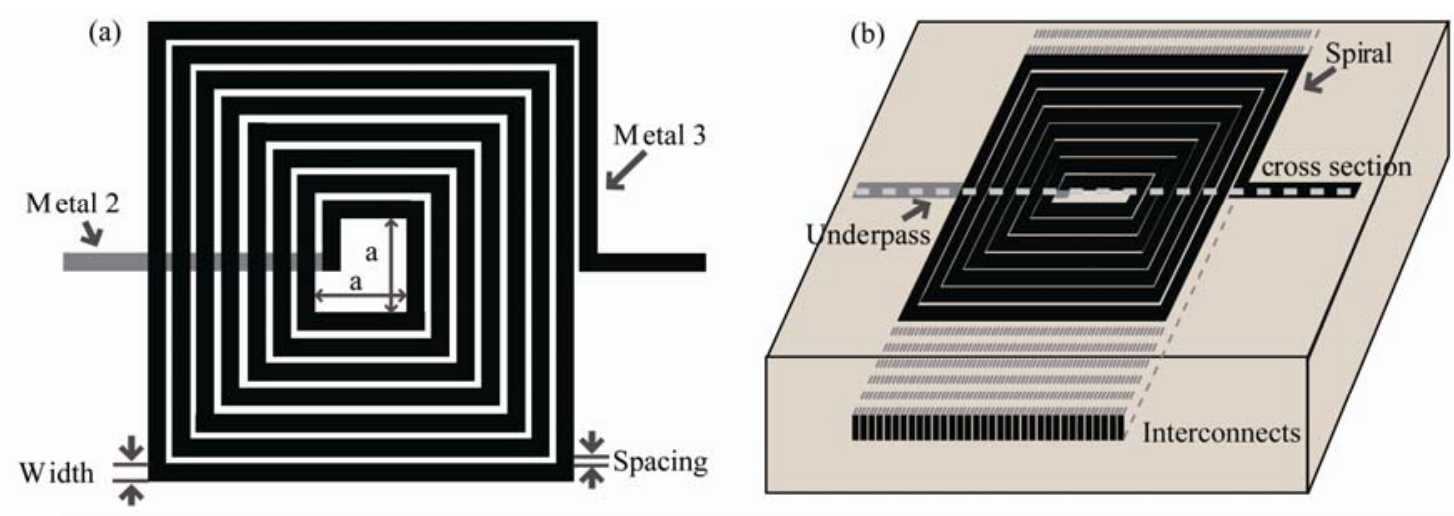

(c)

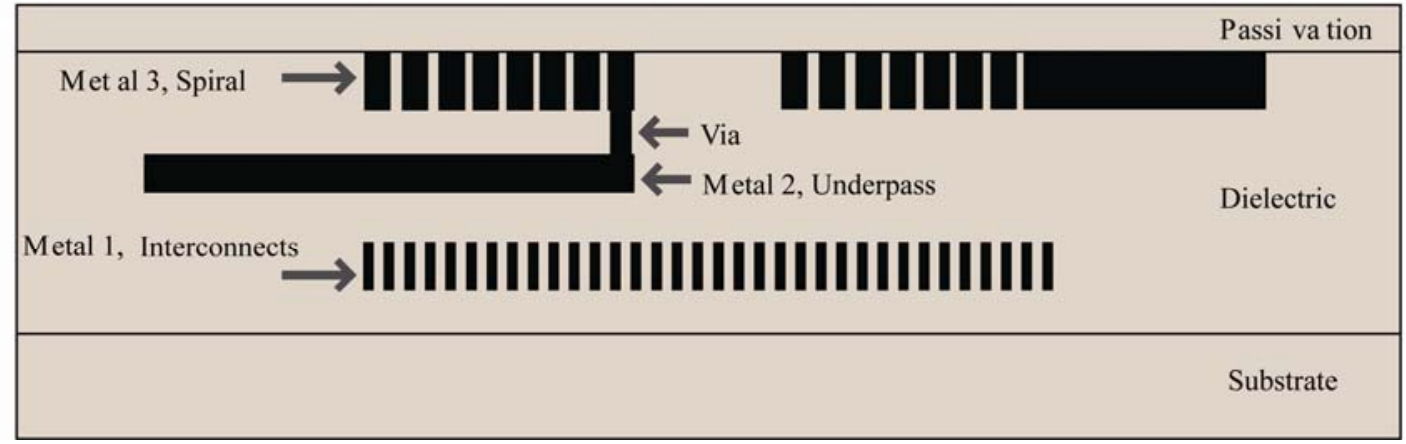

Figure 1. The geometry of the spiral inductor: (a) top layout view of the rectangular spiral; (b) perspective view with metal rods underneath the spiral; (c) cross section of the structure. 


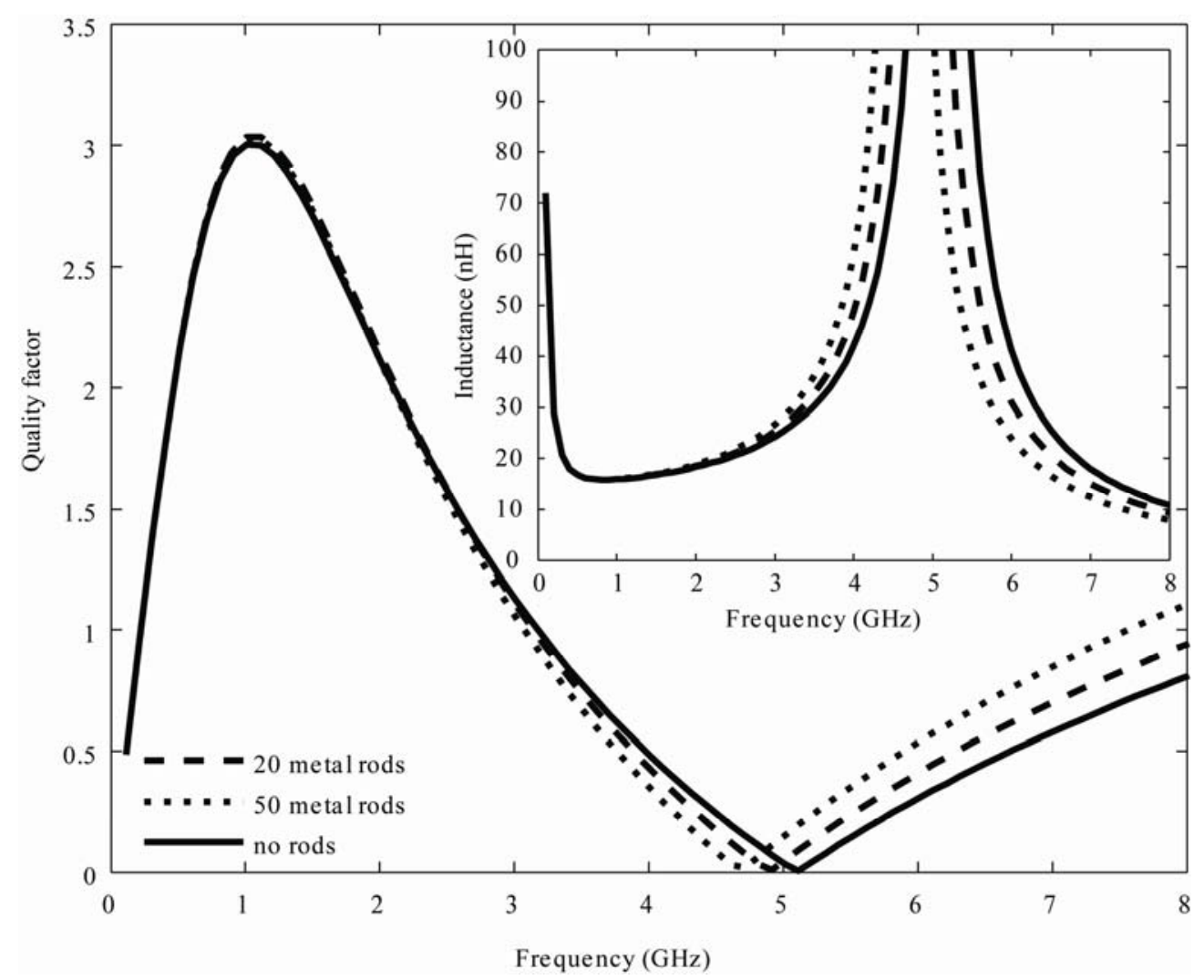

Figure 2. The quality factor and inductance as a function of frequency for rectangular spiral inductor with various numbers of metal rods placed underneath the spiral.

the value and frequency of the maximum Q- factor did not change, but the SRF dropped by $4 \%$ to $4.9 \mathrm{GHz}(9 \%$ to $4.7 \mathrm{GHz}$ ). The more metal rods underneath the spiral, the higher the parasitic capacitance in turn causes lowering of the SRF. The insert plots the inductance and similarly indicates the SRF reduces when rods are present.

Next the rods were replaced with a homogenous equivalent layer of the same thickness. In the empirical fitting procedure the linearly distributed values of the scaling factor $\Psi$ were sampled within the interval of 1 to 10000 with the step increment of 100 . For the particular geometry arrangement denser sampling of the values of $\Psi$ does not reflect in notable change in the characteristic inductor's parameters. It was found that for the studied spiral inductor the fitted value of complex effective permittivity gives good approximation of both the Q-factor and inductance in the considered frequency range of 0.1 $8 \mathrm{GHz}$ when the coefficient $\Psi=7000$ and gives $\varepsilon_{\text {eff }}=$ $28004+7.4069 \times 10^{-3} i$. The results from the EMT simulation of 50 rods are compared against the detailed model in Figure 3, showing good agreement (less than 1\% error). The same accuracy was obtained for the structure with 20 metal rods underneath (not shown here). The value of $\Psi$ is much higher than in the standalone struc- tures $[3,4]$ what is related to the change of the character of illumination wave from the plain wave to the field illuminated by a spiral inductor. Mixtures with conductive constituents can have a larger value of the real part of $\varepsilon_{\text {eff }}$ than either of the components, in certain mixing ratios. Applying the EMT technique gives significant reduction in computational time $(47 \%)$ and allocated memory (45\%) for the case of having 20 metal rods below inductor. This decrease in time and memory consumption approaches $48 \%$ when 50 rods are included and is predicted to decrease further as the complexity of the model increases.

\section{Conclusions}

A numerical study was used to demonstrate that a single layer of interconnects aligned underneath a spiral inductor can be replaced by a homogenous equivalent with effective permittivity calculated based on effective medium theory. The homogenised equivalent structure predicts the values of quality factor and inductance to within $1 \%$. It is also demonstrated that the inductor's performance is affected by the number of conducting rods placed below the spiral. Now that the concept has been demonstrated, future work involves calculating and tabulating 


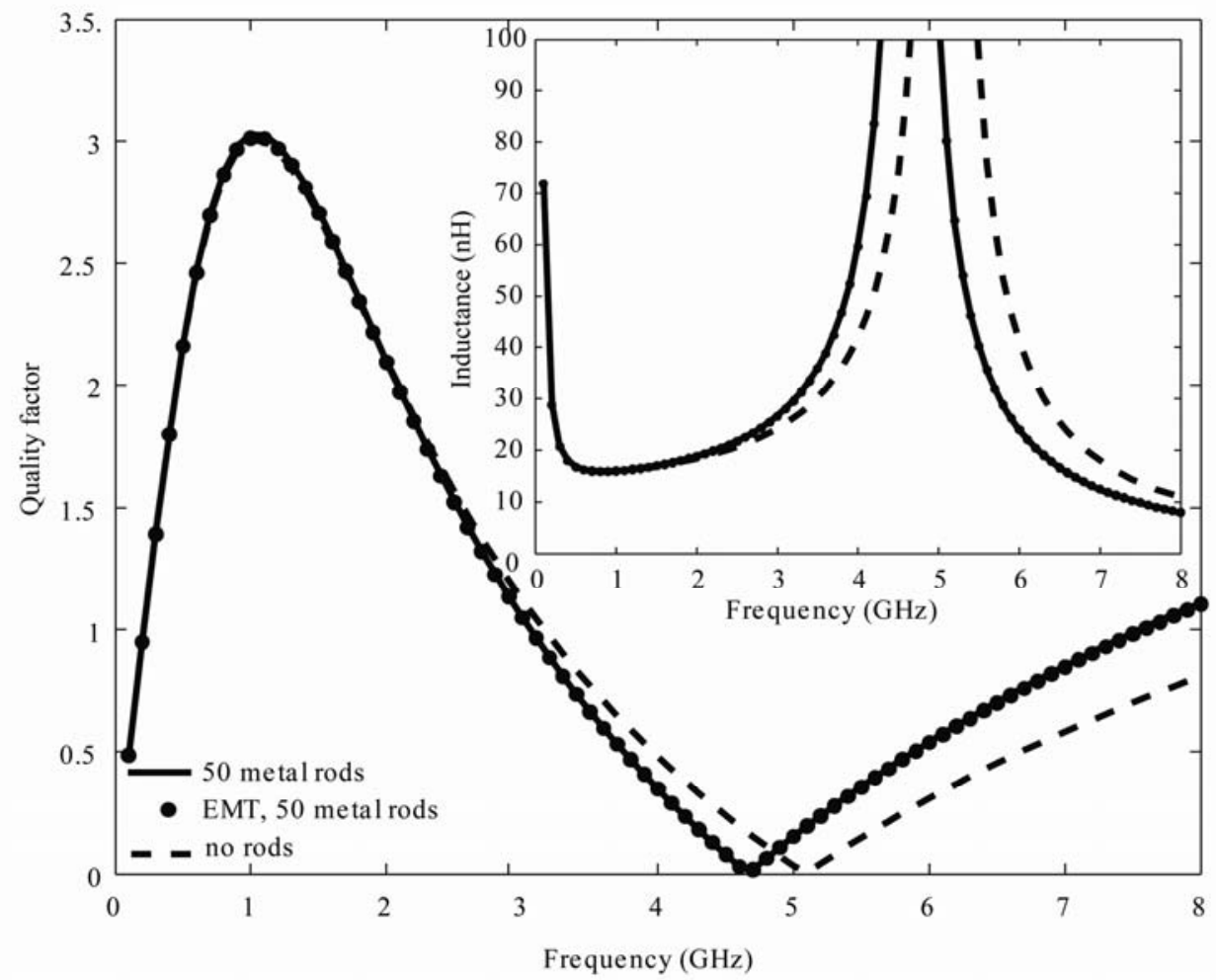

Figure 3. The quality factor and inductance as a function of frequency for rectangular spiral inductor with 50 metal rods placed underneath the spiral. Results from the homogenised equivalent structure are drown as markers.

effective dielectric constant for a range of commonly uncounted geometries.

\section{REFERENCES}

[1] J. Choi, M. Swaminathan, B. Beker and R. Master, "Modeling of Realistic on-Chip Power Grid Using FDTD Method," IEEE International Symposium Electromagnetic Compatibility, Vol. 1, 2002, pp. 238-243.

[2] Fontanelli, "System-in-Package Technology: Opportunities and Challenges," IEEE 9th International Symposium on Quality Electronic Design, 2008, pp. 589-593.

[3] S. M. Holik and T. D. Drysdale, "Effective Medium Approximation for Electromagnetic Compatibility Analysis of Integrated Circuits," Proceedings 2nd International Congress on Advanced Electromagnetic Materials in Microwaves and Optics, 2008, pp. 413-415.

[4] S. M. Holik and T. D. Drysdale, "Simplified Model for On-Chip Interconnects in Electromagnetic Modelling of System-in-Package," Proceedings 12th International Conference on Electromagnetics in Advanced Applications, 2010, pp. 541-544.
[5] J. H. Chang, Y. S. Youn, H. K. Yu, and C. K. Kim, "Effects of Dummy Patterns and Substrate on Spiral Inductors for Sub-Micron RF ICs," IEEE Radio Frequency Integrated Circuits Symposium, 2-4 June 2002, pp. 419-422. doi:10.1109/RFIC.2002.1012081

[6] C. L. Chen, "Effects of CMOS Process Fill Patterns on Spiral Inductors," Microwave and Optical Technology Letters, Vol. 36, No. 6, 2003, pp. 462-465. doi:10.1002/mop. 10790

[7] Ansoft High Frequency Structure Simulator (HFSS), 2010 . http://www.ansoft.com/

[8] M. Park, S. Lee, C. S. Kim, K. H. Yu and K. S. Nam, "The Detailed Analysis of High Q CMOS-Compatible Microwave Spiral Inductors in Silicon Technology," IEEE Transaction Electron Devices, Vol. 45, No. 9, 1998, pp. 1953-1959. doi:10.1109/16.711361

[9] A. H. Sihvola, "Electromagnetic Mixing Formulas and Applications," IEE Publishing, London, 1999. 